\title{
Covid-19 Transmission: Environmental Approach to Effective Control in Closed Indoor Environments
}

\author{
Serge Dolgikh ${ }^{10000-0001-5929-8954] ~}$ \\ Dept. of Information Technology, \\ National Aviation University, Kyiv
}

\begin{abstract}
Closed indoor environments with prolonged contact are known to present heightened risk of Covid-19 transmission. While certain preventative measures have been proposed, they are not yet shown to be very effective. We propose a simple and potentially effective approach to mitigation of these risks in indoor environments based on possible correlation between the characteristics of the environment and the risk of transmission in the droplet and airborne channels.
\end{abstract}

Keywords: Epidemiology, Risk management

\section{Environmental Factors}

Given the possibility of a prolonged coexistence with Covid-19, indicated in some recent studies, intelligent, effective and cost-efficient practices and policies minimizing the risks of infection in multiple scenarios, environments and situations come to the forefront of the society's response to the epidemics. The best effect is achieved when an instrument or a policy is tuned to the specific situation, intelligently, based on the previous practice and strong scientific evidence with continuous evidence gathering, assessment and reassessment, verification of existing and introduction of more effective methods and policies.

The possibility of airborne transmission of Covid-19 indicated by several anecdotal cases [Roosevelt; Office; Case study], has now been advised as established by CDC [CDC]. Based on the analysis of the epidemiological scenarios in the course of 2020 we propose a hypothesis of correlation between the environmental factors such as temperature and humidity and the risk of the transmission. If confirmed, this relationship can be used as a base for a simple, inexpensive and potentially effective technology-based approach to reducing the risks of transmission in the closed higher-risk environments that is measurable and can be replicated throughout straightforwardly with a manageable cost. 


\section{Environmental Factors}

\subsection{Seasonal Patterns in Covid-19 Pandemics}

Covid-19 infections have shown significant reduction during the summer months of 2020 across multiple jurisdictions in Europe. This effect was at first attributed to the success of the epidemiological policies, but subsequent development in the fall of 2020 showed it not to be the case. As the temperature and humidity in the northern hemisphere moved in the opposite directions, the rate of transmission of Covid-19 started rising again, at an alarming in many jurisdictions, rates [5].

Let's note that the summer of 2020 was also recorded as one of the hottest and driest in the history.

A straightforward deduction from these observations then leads to the hypothesis that high temperature combined with low humidity naturally suppresses both droplet and airborne transmission channels possibly via faster evaporation of the transmission media such as droplets or aerosols.

\subsection{Covid-19 Transmission Suppression in Closed Indoor Environments: Method and Technology}

Prolonged presence in closed indoor environments is known to represent higher risk of transmission and the confirmation of the possibility of airborne transmission goes a long way in explaining anecdotal cases of transmission in such environments as offices; churches; residences; work places; public transport and others [6-13] even where common preventative measures were taken.

If the environmental factor correlation is confirmed by further research and verification, a simple method can be proposed to suppress transmission channels in the closed indoor environments by managing environmental factors such as: temperature; humidity; and air flow.

\section{Temperature:}

Higher temperature settings up to mid or even higher 30 -ies $\mathrm{C}$, as recorded in summer of 2020.

\section{Humidity:}

Reduced humidity, with dry and warm air throughout the closed environment

Airflow:

Change the patterns of airflow by modifying ventilation system; rather than circulating the air from a single or a small number of sources, with the sink and sources on the same level creating complex airflows with possibility of accumulation of viral load at certain points, a vertical airflow can be suggested with multiple sources and sinks, removing the exhaled air upwards outside of populated areas and immediately disposing it outside. 

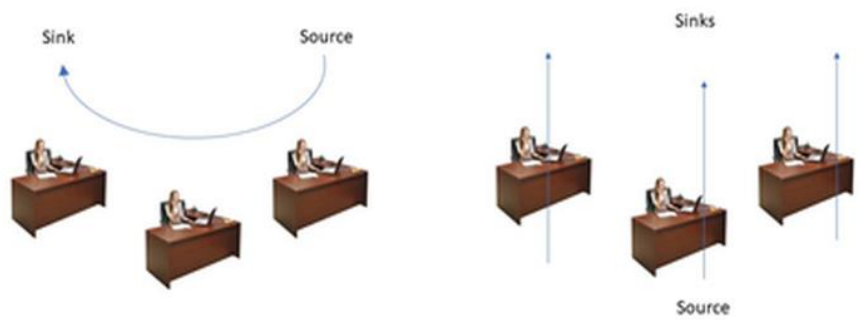

Fig.1 Transmission suppressing airflow design

Controlling environmental factors in the managed environments may allow to influence two important transmission factors: 1) the rate of evaporation of exhaled media such as droplets and aerosols; and 2) the pattern of its distribution in the closed space.

Such an approach while straightforward and cost-effective, may be able to significantly reduce the risk of transmission from a remote source of infection via airflow in the indoor space that may not be easily detectable with common contact tracing.

\subsection{Contrary Cases}

In many reported cases Covid-19 epidemics peaked during summer months including Brazil, Mexico, India and south of USA resulting in high number of cases and severe impact. Some factors may explain it as possibly not contradicting the environment correlation hypothesis:

- High humidity in tropical and near locations

- Higher density of population

- Culture of close and frequent inter-personal contact

- Less effective control policies

Nevertheless, it is clear that further research and experimentation needs to be done to confirm the validity of the hypothesis.

\subsection{Distancing and Common Policies}

Distancing and other policies should be maintained to maximize the effect of suppression of infection transmission in the closed environments. Other technology-based methods and approaches [14] can be implemented to maximize the effect.

\section{Conclusion}

The proposed technology-based method of suppressing transmission of Covid-19 in the higher-risk closed indoor environments via control of environmental factors are straightforward and inexpensive. If the validity of the underlying hypothesis is confirmed by subsequent research, the method can provide an effective, cost efficient and versatile instrument in controlling the epidemics in the environments that represented a challenge to control and higher risk of transmission. 


\section{References}

1. [Roosevelt] Dyer A., CDC study of Roosevelt outbreak finds lower rates of COVID-19 among sailors who wore masks, San Diego Union-Tribune, June 2020.

https://www.sandiegouniontribune.com/news/military/story/2020-06-09/cdc-study-ofroosevelt-outbreak-finds-lower-rates-of-covid-19-among-sailors-who-wore-masks.

2. [Office] https://www.erinbromage.com/post/the-risks-know-them-avoid-them.

3. [Case study] Covid-19-Case-Cluster-Study: https://www.deutschlandfunk.de/heinsbergstudie-und-andere-zum-coronavirus-wo-sich-die.2897.de.html?dram:article_id=474889

4. [CDC] Advisory on Covid-19 airborne transmission https://www.cdc.gov/media/releases/2020/s1005-how-spread-covd.html 5.10.2020.

5. Coronavirus map Google https://www.google.com/covid19-map/ (6.10.2020).

6. Church outbreak, Global News https://globalnews.ca/news/7382376/lethbridge-spikecovid-19-cases/ 6.10.2020.

7. Senior's homes, Quebec: https://montreal.ctvnews.ca/covid-19-continues-to-ravagequebec-seniors-homes-1.4902957.

8. Half of coronavirus deaths happen in care homes data from EU suggests: Guardian, https://www.theguardian.com/world/2020/apr/13/half-of-coronavirus-deaths-happen-incare-homes-data-from-eu-suggests.

9. Covid-19 outbreak, meatpacking plant, Alberta: https://www.cbc.ca/news/canada/calgary/cargill-high-river-jbs-brooks-deena-hinshaw1.5545113 .

10. University residence, New York: https://www.nbcnewyork.com/news/local/studentsshocked-confused-amid-urgent-move-out-order-at-nyc-residency-hit-with-covid-19death/2341955/.

11. Dormitory Covid-19 clusters: Singapore, Ukraine https://www.straitstimes.com/singapore/coronavirus-new-facilities-will-be-built-to-housemildly-ill-and-recovering-workers-in; https://en.interfax.com.ua/news/general/659350.html.

12. What the cruise-ship outbreaks reveal about COVID-19: Nature, https://www.nature.com/articles/d41586-020-00885-w.

13. OC Transpo drivers report cases of Covid-19 https://theworldnews.net/ca-news/octranspo-driver-tests-positive-for-covid-19 10.8.2020.

14. Dolgikh, S. Smart-Covid: Intelligent solutions for higher risk environments, preprint ResearchGate doi: 10.13140/RG.2.2.12754.38088, August 2020. 